\title{
INVESTIGACIÓN
}

Recibido: 20/01/2021 --- Aceptado: 16/03/2021 --- Publicado: 10/05/2021

\section{EL «EFECTO VENTRILOCUO» EN LAS AGENCIAS INTERNACIONALES DE NOTICIAS. REVISIÓN TEÓRICA E INCIDENCIA EN LAS NUEVAS FORMAS DE DESINFORMACIÓN}

\author{
The «ventrilocuo effect» of the international news agencies. Theoretical review \\ and incidence on new forms of misinformation
}

(D) $8 \mathbb{R}^{6}$ Sabina Civila1. Universidad de Huelva. España.

sabicivila@gmail.com

(D) 8) ${ }^{\mathrm{R}}$ Bárbara Castillo-Abdul. Universidad Rey Juan Carlos. España.

barbaracastilloabdul@gmail.com

(i) $8 R^{a}$ Luis Miguel Romero-Rodríguez. Universidad Rey Juan Carlos. España.

Lromero2021@gmail.com

Este trabajo está avalado por el Proyecto I+D+I (2019-2021), titulado "Youtubers e Instagramers: La competencia mediática en los prosumidores emergentes" con clave RTI2018-093303-B-I00, financiado por Ministerio de Ciencia, Innovación y Universidades de España y el Fondo Europeo de Desarrollo Regional (FEDER) y por el Proyecto I+D-i (2020-2022), titulado "Instagramers y youtubers para el empoderamiento transmedia de la ciudadanía andaluza. La competencia mediática de los instatubers", con clave P18-RT-756, financiado por la Junta de Andalucía, en la convocatoria 2018 (Plan Andaluz de Investigación, Desarrollo e Innovación, 2020) y el Fondo Europeo de Desarrollo Regional (FEDER).

\section{Cómo citar el artículo:}

Civila, S., Castillo-Abdul, B., y Romero-Rodríguez, L. M. (2021). El «efecto ventrilocuo» en las agencias internacionales de noticias. Revisión teórica e incidencia en las nuevas formas de desinformación. Vivat Academia. Revista de Comunicación, 154, 25-46. http://doi.org/10.15178/va.2021.154.e1302

http://www.vivatacademia.net/index.php/vivat/article/view/1302

\section{RESUMEN}

Esta investigación reflexiona, desde una perspectiva teórica, cómo a través de las agencias de noticias se difunden la mayoría de los acontecimientos internacionales que llegan al resto de los medios de comunicación, provocando el conocido «efecto

\footnotetext{
1Sabina Civila: Investigadora Predoctoral FPI en el Departamento de Pedagogía de la Universidad de Huelva. Doctoranda en el programa Interuniversitario de Comunicación (UHU) en la línea de Educomunicación.
} 
Civila, S., Castillo-Abdul, B., y Romero-Rodríguez, L. M.

El «efecto ventrilocuo» en las agencias internacionales de noticias. Revisión teórica e incidencia en las nuevas formas de desinformación

ventrílocuo»: múltiples medios, una sola voz; y se indaga sobre cómo las plataformas online han fomentado este fenómeno, provocando un aumento estructural de la desinformación. En este sentido, la investigación tiene por objetivo conocer el desarrollo del «efecto ventrílocuo» con el progreso de los «nuevos medios» y como consecuencia, el aumento de la desinformación. Para este análisis documental de teoría fundamentada, el procedimiento metodológico se ha basado en la revisión bibliográfica de la literatura en las bases de datos internacionales de referencia (WoS y Scopus), realizando un análisis de estudios primarios, con el fin de sintetizar la información. Los resultados indican, entre otras cuestiones, que las redes sociales fomentan espacios de desinformación estructural en el ecosistema actual. En conclusión, se determina la relación entre el «efecto ventrílocuo» y la desinformación, que surge como consecuencia de la reticularidad y descentralización informativodigital.

PALABRAS CLAVE: Redes sociales- medios digitales - comunicación - efecto ventrílocuo - desinformación - era digital -participación ciudadana - prensa digital educomunicación.

\section{ABSTRACT}

This research reflects, from a theoretical perspective, how most of the international events that reach the rest of the media are disseminated through news agencies, causing the well-known «ventriloquist effect»: multiple media, a single voice; and explores how online platforms have fostered this phenomenon, causing a structural increase in misinformation. In this sense, the research aims to understand the development of the «ventriloquist effect» with the progress of «new media» and, as a consequence, the increase of disinformation. For this grounded theory documentary analysis, the methodological procedure was based on the bibliographic review of the literature in the international reference databases (WoS and Scopus), carrying out an analysis of primary studies to synthesize the information. The results indicate, among other issues, that social networks foster spaces of structural misinformation in the current ecosystem. In conclusion, the relationship between the "ventriloquist effect" and misinformation, which arises from reticularly and information-digital decentralization, is determined.

KEYWORDS: Social networks - digital media - communication - ventriloquist effect fake news - digital age - citizen participation - digital press - media literacy.

\section{O <<EFEITO VENTRÍLOQUO > NAS AGÊNCIAS INTERNACIONAIS DE NOTÍCIAS. REVISÃO TEÓRICA E INCIDÊNCIA NAS NOVAS FORMAS DE DESINFORMAÇÃO.}

\section{RESUMO}

Esta pesquisa reflexiona, desde uma perspectiva teórica, como através das agências de notícias se difundem a maioria dos acontecimentos internacionais que chegam ao resto 
Civila, S., Castillo-Abdul, B., y Romero-Rodríguez, L. M.

El «efecto ventrilocuo» en las agencias internacionales de noticias. Revisión teórica e incidencia en las nuevas formas de desinformación

dos meios de comunicação, provocando o conhecido <<efeito ventríloquo>>: vários meios de comunicação, somente uma voz, e se investiga sobre como as plataformas online fomentam este fenômeno, causando um aumento estrutural da desinformação. Neste sentido, a pesquisa tem por objetivo conhecer o desenvolvimento do $<<$ efeito ventríloquo $>>$ com o crescimento dos $<<$ novos meios de comunicação $>>$ e como consequência, o aumento da desinformação. Para esta análise documental de teoria fundamentada, o procedimento metodológico foi a revisão bibliográfica da literatura com as bases internacionais de referência(Wos e Scopus), fazendo uma análise de estudos primários, com a finalidade de sintetizar a informação. Os resultados indicam, entre outras questões, que as redes sociais fomentam espaços de desinformação estrutural no ecossistema atual. Como conclusão, se determina a relação entre o «efeito ventríloquo» e a desinformação que surge como consequência da reticularidade e descentralização informativa-digital.

\section{PALAVRAS CHAVE}

Redes sociais - meios digitais - comunicação - efeito ventríloquo - desinformação - era digital - participação cidadã - imprensa digital - educomunicação.

\section{INTRODUCCIÓN}

Las agencias de noticias son empresas dedicadas a la elaboración de informaciones y a la comercialización de estos (Bustor y Pastor, 1995). Partiendo de esta premisa, se puede afirmar que el espectro de la pluralidad queda limitado, dando paso a lo que se conoce como el «efecto ventrílocuo»: "múltiples medios, una sola voz" (Arráez, 1998). Es decir, que, a pesar de existir una gran cantidad de medios de comunicación, la mayoría de la información internacional proviene de las mismas fuentes, bien sea por una reducción de corresponsalías propias, o por la propia imposibilidad de los medios de tener periodistas ubicuos.

Como consecuencia, las informaciones enviadas al lector quedan debilitadas por tres aspectos: (1) sesgo etnocéntrico, (2) manejo de otras fuentes y, (3) el efecto agenda setting. Con el surgimiento y auge de Internet y, por tanto, periódicos digitales y redes sociales, el «efecto ventrílocuo» ha aumentado su alcance e impacto, lo que ha generado consecuencias como la expansión de los sesgos. Estos conceptos serán objeto de estudio en el texto, reflexionando sobre la relación entre la derivación ventrílocuo y la desinformación por sesgos en la era de la información.

En este sentido, se realiza una investigación documental que inicia con la revisión de los estudios y literatura académica reciente, que permite encuadrar la investigación, fundamentar el campo de estudio y comprender las consecuencias derivadas del «efecto ventrílocuo» en el ecosistema digital. Para ello se ha realizado una exploración por las bases de datos WoS y Scopus, bajo el término de búsqueda desinformación (misinformation) y (booleano AND) agencias de noticias (news agencies). Una vez obtenido el listado primario (nSUM=11274), se limitó la búsqueda solo a las revistas de ciencias sociales desde 2015 hasta 2020 por criterios de actualidad temática, emergiendo un total de 2089 documentos. Una vez realizada la primera fase, se llevó

Vivat Academia. Revista de Comunicación. 2021, nº 154, 25-46 
Civila, S., Castillo-Abdul, B., y Romero-Rodríguez, L. M.

$E l$ «efecto ventrilocuo» en las agencias internacionales de noticias. Revisión teórica e incidencia en las nuevas formas de desinformación

a cabo una segunda criba de revisión de la literatura y de teoría fundamentada, con las que se correlacionarían las mismas formas epistemológicas, ontológicas y teóricas. Todo ello, a través del análisis de contenido de base interpretativa.

Los resultados obtenidos indican cómo el «efecto ventrílocuo» constituye una idea dominante, escondida bajo una supuesta pluralidad que en realidad no es más que la paráfrasis de diferentes agencias y que se ve agravado por el alcance que pueden tener los medios de comunicación online y la falta de alfabetización mediática de las audiencias, con respecto a criterios de interpretación informativa y comprensión de sesgos.

Así, el objetivo principal de la presente investigación es conocer el desarrollo teórico e impacto en la praxis comunicativa del «efecto ventrílocuo» en relación con el progreso de los nuevos medios, así como identificar el aumento de la desinformación por sesgos como consecuencia de la reticularidad del ecosistema digital, surgiendo así la pregunta principal de investigación (RQ1) ¿Cómo influye la evolución digital en la desinformación y el efecto ventrílocuo?

\section{MATERIALES Y MÉTODO}

La presente investigación tiene un carácter exploratorio y descriptivo, estableciendo como campo de análisis el impacto de las noticias procedentes de una misma fuente (agencia internacional de noticias) y la relevancia que adquiere la desinformación por sesgos con este fenómeno en el ámbito digital. Para alcanzar los objetivos propuestos se realiza una revisión de la literatura actual (2015-2020) sobre los medios digitales y la desinformación. El desarrollo del estudio se estructura en tres fases: (i) Diseñar la estrategia de búsqueda, (ii) la recopilación de la literatura especializada en las nuevas formas de desinformación y, (iii) el análisis de la información extraída.

Para diseñar la estrategia de búsqueda se identificaron las palabras clave que guardan relación con el objeto de estudio, lo que permite delimitar el número de artículos resultantes de la búsqueda preliminar. Una vez se realizó el criterio de búsqueda "desinformación" (misinformation), las palabras seleccionadas fueron: desinformación, medios digitales, agencias de noticias, «efecto ventrílocuo», redes sociales y era digital. Es decir, tras la primera exploración inicial, se seleccionaron aquellos documentos en los que surgían las palabras anteriores.

El motivo por el que se selecciona como objeto de búsqueda "desinformación" en vez de "ventriloquism ", es porque al realizar la búsqueda solo por efecto ventrílocuo (ventriloquism), la gran parte de literatura resultante hace relación a los estudios sobre ventriloquia (arte para modificar e imitar voces y sonidos), dado que el término, si bien se utiliza desde hace más de una década, no está universalizado en la comunidad científica de la comunicación y el periodismo.

Esta revisión documental se realizó en bases de datos científicas, siguiendo un orden por impacto, comenzando por la colección principal de la Web of Science (WoS;

Vivat Academia. Revista de Comunicación. 2021, n 154, 25-46 
Civila, S., Castillo-Abdul, B., y Romero-Rodríguez, L. M.

$E l$ «efecto ventrilocuo» en las agencias internacionales de noticias. Revisión teórica e incidencia en las nuevas formas de desinformación

seguido por Scopus. Se escogen estas dos bases de datos al ser de carácter internacional y con procedimientos selectivos fundamentados en impactos cuantitativos (citas), entendiendo que los documentos emergentes en estos repositorios han tenido un control previo, revisión por pares y visibilidad científica.

Se realizó una exploración seleccionando el período 2015-2020 para dotar a la investigación de un encuadre novedoso y detectar el desarrollo actual de la literatura sobre esta temática en los últimos cinco años (ver figura 1). Para llevar a cabo la búsqueda se tuvieron en cuenta los siguientes criterios de selección:

a) Relación temática: Se analizaron en Scopus 6525 documentos y en WoS 4749, refinándose a solo aquellos relacionados con el objeto de estudio (área Ciencias Sociales-Comunicación, tipo de documento artículo y fuente revista). Después de esta criba, quedan seleccionados 1044 en Scopus y 1049 en WoS.

b) Por la novedad del artículo: Para ello, se limitó la búsqueda a los años 2015-2020 y se tomaron en cuenta el número de citas emergentes en WoS y Scopus, con especial énfasis en los más novedosos. El ámbito geográfico no se delimita, considerándose todos los documentos emergentes, tras la criba, independientemente de su origen.

c) Por su impacto cuantitativo: Se tomaron en cuenta los más citados (organización). Emergen 18 documentos, de los cuales solo se seleccionan aquellos con más de 20 citas. Si bien es cierto que los artículos más novedosos (por ejemplo 2018-2020) son menos propensos a tener este número de citas tan elevados para el área, el criterio de citaciones es importante porque demuestra que el artículo se ha convertido en referencia de investigación para los académicos.

En Scopus se realizó el criterio de búsqueda desinformación (misinformation). Siguiendo la misma dinámica que para WoS, para el refinado de pesquisa se optó por la selección de documentos emergentes 2015-2020, del área de Ciencias Sociales, tipo de documento artículo y tipo de fuente revistas. Antes de la primera criba emergieron 6.525 documentos, que después del refinado se convirtieron en 1044. Por su parte, en la Web of Science (WoS) se aplicaron los mismos criterios, reflejando en el primer filtrado 4.749 documentos. Para el filtrado, se seleccionó el período 2015-2020, solo en el ámbito temático de la Comunicación [Communication], tipo de documento artículo, obteniéndose de esta criba 1.049 documentos (ver figura 1). 
Civila, S., Castillo-Abdul, B., y Romero-Rodríguez, L. M.

El «efecto ventrilocuo» en las agencias internacionales de noticias. Revisión teórica e incidencia en las nuevas formas de desinformación

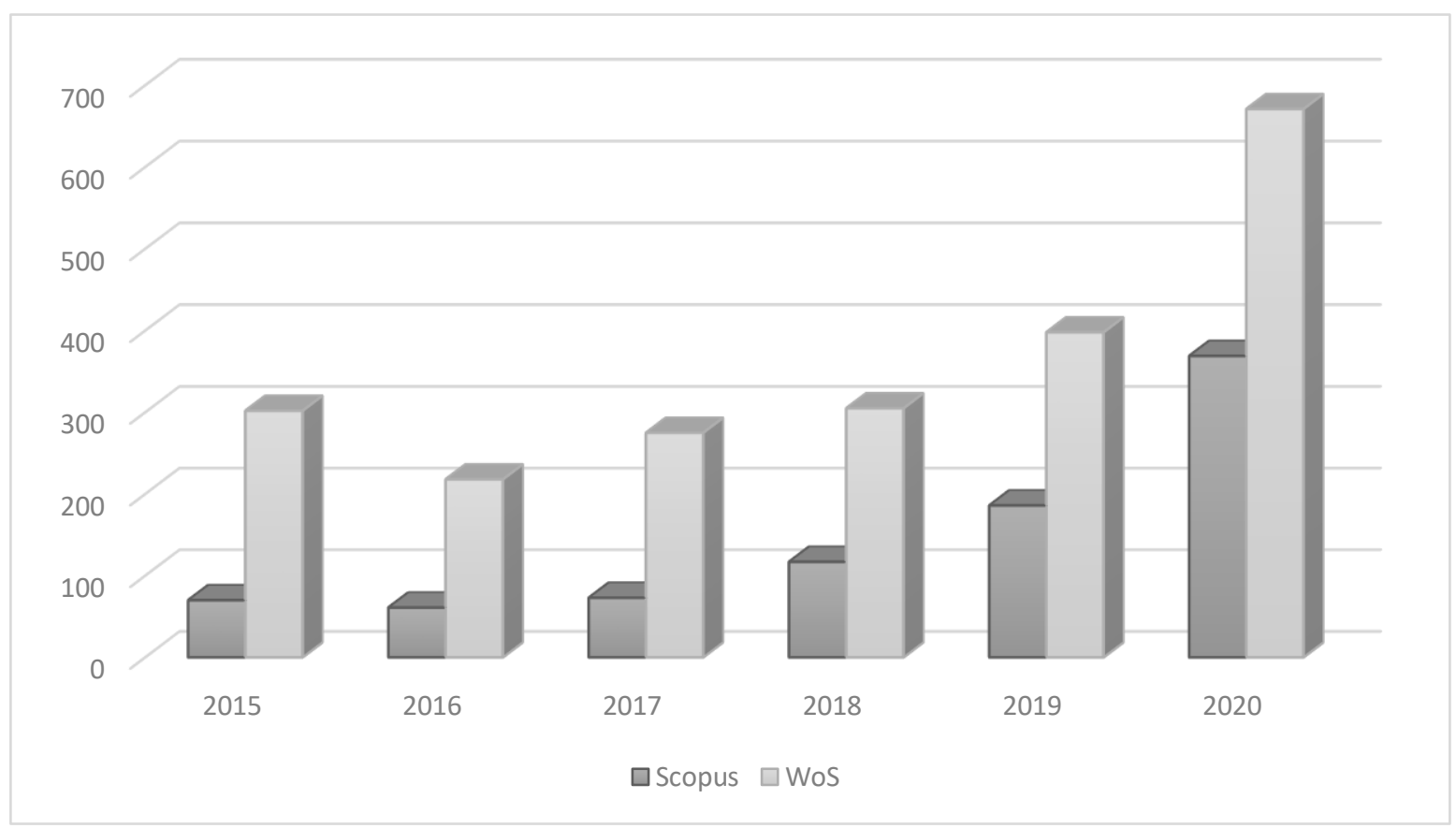

Figura 1. Documentos emergentes en Wos y Scopus (2015 - 2020)

Una vez se realizó el primer cribado, se seleccionaron aquellas investigaciones que incluían las palabras clave: desinformación, medios digitales, agencias de noticias, «efecto ventrílocuo», redes sociales y era digital en el «tema» o los metadatos (título, resumen y/o palabras clave). Con estos documentos se llevó a cabo una revisión de la literatura y de teoría fundamentada con las que se correlacionarían las mismas formas epistemológicas, ontológicas y teóricas.

El análisis ha consistido en una lectura objetiva y exhaustiva de los documentos resultantes de los criterios de selección utilizados, a través de un mapping o matriz de revisión, para llegar a la literatura más relevante extrayendo aquella información referente a nuestros criterios de búsqueda y que nos conducen hasta los objetivos planteados. Para llevar a cabo este análisis de datos, el procedimiento utilizado se ha basado en el análisis de contenido de base interpretativa, justificado como instrumento cualitativo de recogida de información.

\section{RESULTADOS}

\subsection{Las agencias de noticias}

Las agencias de noticias son el medio de comunicación menos estudiado en Ciencias Sociales, producto de su origen, el servicio a otros medios de comunicación, ejerciendo de intermediarias entre los acontecimientos y las empresas informativas (Jiménez, 2011). En esta línea, De Bustos y Pastor (1995, p. 21) afirman que "las agencias de noticias son las empresas que se dedican principalmente a la elaboración de todo tipo 
Civila, S., Castillo-Abdul, B., y Romero-Rodríguez, L. M.

$E l$ «efecto ventrilocuo» en las agencias internacionales de noticias. Revisión teórica e incidencia en las nuevas formas de desinformación

de información para venderlas posteriormente". Jiménez (2011), a raíz de la clasificación de los referidos autores, y de acuerdo con diversas funciones, realiza una clasificación más actual (tabla 1):

Tabla 1. Clasificación de las agencias internacionales de noticias

\begin{tabular}{|c|c|c|c|c|c|}
\hline \multicolumn{2}{|c|}{ Por ámbito geográfico } & \multicolumn{2}{|c|}{ Por tipo de información } & \multicolumn{2}{|c|}{ Por propiedad } \\
\hline 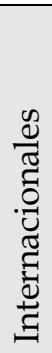 & $\begin{array}{l}\text { Son aquellas que } \\
\text { comenzaron siendo agencias } \\
\text { nacionales y dieron el salto a } \\
\text { la internacionalización, } \\
\text { contratando periodistas en el } \\
\text { extranjero para } \\
\text { comercializar sus contenidos } \\
\text { fuera del país }\end{array}$ & 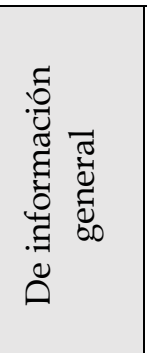 & $\begin{array}{l}\text { Son aquellas que informan sobre } \\
\text { cualquier tipo de acontecimiento }\end{array}$ & 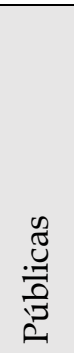 & 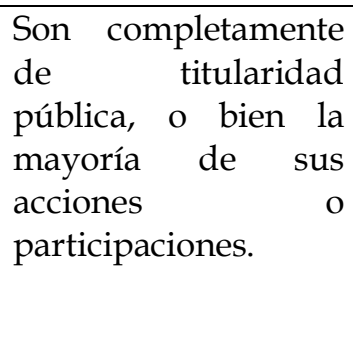 \\
\hline 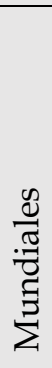 & $\begin{array}{l}\text { Son aquellas que tienen } \\
\text { cobertura en todos los } \\
\text { países. Tienen un amplio } \\
\text { número de corresponsales y, } \\
\text { por tanto, tienen mayor } \\
\text { posibilidad de obtener } \\
\text { hechos noticiables. }\end{array}$ & 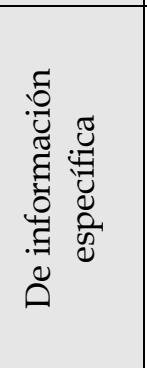 & $\begin{array}{l}\text { Centran su actividad } \\
\text { informaciones concretas. }\end{array}$ & \multirow{6}{*}{ 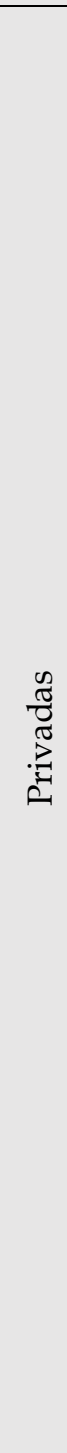 } & \multirow[t]{6}{*}{$\begin{array}{l}\text { Son aquellas cuya } \\
\text { propiedad pertenece } \\
\text { a una persona física o } \\
\text { jurídica de naturaleza } \\
\text { privada. }\end{array}$} \\
\hline 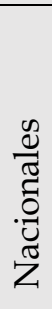 & $\begin{array}{l}\text { Según la definición de la } \\
\text { UNESCO de 1953, estas } \\
\text { agencias son aquellas que } \\
\text { solo recogen noticias } \\
\text { nacionales y las distribuyen } \\
\text { en aquellos países donde } \\
\text { tienen sede. }\end{array}$ & 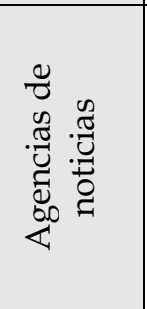 & $\begin{array}{l}\text { Encargadas de enviar a los } \\
\text { medios de comunicación } \\
\text { noticias, crónicas, reportajes, } \\
\text { entrevistas. }\end{array}$ & & \\
\hline \multirow{4}{*}{ 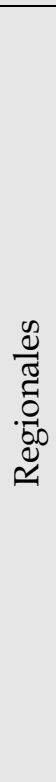 } & \multirow{4}{*}{$\begin{array}{l}\text { Dos elementos son } \\
\text { indispensables para } \\
\text { considerar una agencia } \\
\text { regional, de acuerdo con } \\
\text { Jiménez (2011): Primero, la } \\
\text { mayor parte del capital debe } \\
\text { pertenecer a entidades, } \\
\text { accionistas o participantes } \\
\text { de la propia comunidad. Por } \\
\text { otro lado, las noticias de este } \\
\text { medio deben estar enfocadas } \\
\text { a la comunidad regional o } \\
\text { local. }\end{array}$} & 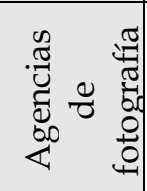 & $\begin{array}{l}\text { Ofrecen elementos fotográficos y } \\
\text { visuales. }\end{array}$ & & \\
\hline & & 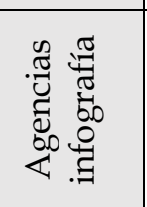 & $\begin{array}{l}\text { Se encargan de distribuir a los } \\
\text { medios infografías y gráficos. }\end{array}$ & & \\
\hline & & 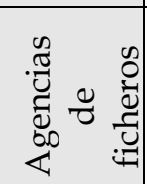 & $\begin{array}{l}\text { Aportan cortes y documentación } \\
\text { que han sido resultado de } \\
\text { entrevistas o programas } \\
\text { anteriores. }\end{array}$ & & \\
\hline & & 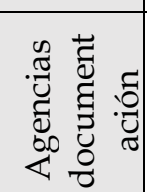 & $\begin{array}{l}\text { Están especializadas en la } \\
\text { elaboración de datos para su } \\
\text { venta a los medios de } \\
\text { comunicación. }\end{array}$ & & \\
\hline
\end{tabular}

Fuente: Elaboración propia a partir de De Bustos y Pastor (1995) y Jiménez (2011) 
Civila, S., Castillo-Abdul, B., y Romero-Rodríguez, L. M.

$E l$ «efecto ventrilocuo» en las agencias internacionales de noticias. Revisión teórica e incidencia en las nuevas formas de desinformación

La batalla de las agencias por la cobertura de las noticias internacionales se libra fundamentalmente entre cuatro grandes casas: Associated Press, Reuters, France Press y la nacional EFE (Gelado, 2009).

Cualquier profesional de los medios o estudioso de la comunicación sabe que el rol de las agencias de prensa en el panorama mediático español y mundial es mucho más importante de lo que trasciende al gran público. Sin ellas sería difícilmente entendible la multiplicidad informativa y de entretenimiento al que se ha visto sometida la comunicación de masas en los últimos años (Frost, 2019). Con todo, el hecho de que hayan ofrecido desde su nacimiento hasta su poderoso presente un ejercicio tal, no quiere decir que no hayan traído un buen número de consecuencias de dudosa deseabilidad (Gelado, 2009).

Un ejemplo de consecuencia negativa por el uso indiscriminado de las agencias de noticias es que la mayor parte de los grandes periódicos españoles y extranjeros se alimentan de varias agencias de noticias, pero ninguna de ellas se sale del ámbito occidental (el ejemplo podría extrapolarse allende nuestras fronteras con grandes probabilidades de éxito); por lo que el sesgo etnocéntrico está garantizado (Gelado, 2009). Como asegura Muro Benayas (2006, p. 22):

[...] aunque la imparcialidad y el rigor son atributos que forman parte del imaginario de todas las agencias, su práctica está asociada a una determinada cosmovisión -anglosajona, árabe, latinoamericana o asiática- que planea por encima de la neutralidad de sus redactores.

Todo ello, en la práctica, se traduce no sólo en un volumen de noticias muy inferior para determinados puntos del planeta, lo cual podría justificarse por el criterio de proximidad, sino también en la creación de ciertos estereotipos referentes a otras culturas, al tiempo que redunda en la dificultad de elaborar un retrato honesto de según qué países y culturas con el escaso número de noticias que se les dedican (Gelado, 2009).

Chomsky y Herman (1990, p. 69) afirman:

el modelo de los medios de comunicación masiva actúa como activo propagandístico a través de varios filtros, entre los que destacan: 1) el tamaño, la concentración de propiedad y orientación de las empresas dominantes, 2) la publicidad como fuente de ingresos, 3) la dependencia de los medios hacia el gobierno y/o las empresas y, 4) la acción de los grupos de presión sobre los periodistas.

De igual modo, las noticias que provienen de las agencias y medios de comunicación internacionales tienen cierta influencia en las impresiones y actitudes de las personas que reciben el mensaje hacia el país al que se refieren (Camacho Domínguez, Romero-Rodríguez y Aguaded, 2018), sobre todo porque la mayor parte de las audiencias no tienen acceso a la pluralidad informativa de los eventos internacionales (Wu, 1998; López-García, 2006; Torres-Toukoumidis et al., 2017.

Vivat Academia. Revista de Comunicación. 2021, nº 154, 25-46 
Civila, S., Castillo-Abdul, B., y Romero-Rodríguez, L. M.

El «efecto ventrilocuo» en las agencias internacionales de noticias. Revisión teórica e incidencia en las nuevas formas de desinformación

En última instancia, el único modo de neutralizar el efecto de sesgo de las agencias sería, o bien multiplicar su número, diversificar su procedencia o hacer que más medios domésticos apostaran por ser completamente independientes de su influjo, aunque ello implicase salirse de la obsesión por la inmediatez en la que la gran mayoría vive sumida desde hace ya algún tiempo (Gelado, 2009).

\subsection{El «efecto ventrílocuo»}

Tras profundizar acerca de las agencias de noticias y entender cuáles son sus labores más significativas, se comprende que estas organizaciones fijan fácilmente la naturaleza del público internacional, así como de los asuntos públicos y su tratamiento (priming, framing y gate keeping) con consecuencias como la espiral del silencio (Day, 2018) o el efecto bandwagon. Esto se debe a que tanto los periodistas, como el público, confían en ellos básicamente porque dominan los canales de distribución de noticias (Tunstall, 2008), es decir, su credibilidad está sustentada en los propios cimientos de su ejercicio (Rivera-Rogel et al., 2017; Rodríguez-Hidalgo, Rivera-Rogel y RomeroRodríguez, 2020).

El «efecto ventrílocuo» de acuerdo con Arraez (1998) se define como "múltiples medios, una sola voz", es decir, como una selección de noticias que se emiten a través de múltiples medios de comunicación, pero que provienen de una misma agencia (parafraseada o no). El avance de los estudios sobre el «efecto ventrílocuo» ha llevado a otros autores a generar nuevas definiciones como la ofrecida por TorresToukoumidis et al., (2017) o Tejedor-Calvo et al. (2020) quienes defienden que este efecto consiste en perspectivas informativas que se asientan en la opinión pública debido a la falta de capacidad de los medios de generar otros encuadres (frames). Con el desarrollo de las tecnologías se cuestiona el concepto y surgen nuevas ideas como la propuesta por Day (2018), que define el «efecto ventrílocuo» como dominio político, económico y cultural que ha estado en continuo crecimiento y se encuentra en crisis por el surgimiento de nuevas voces digitales. Asimismo, en la misma línea Frost (2019) expone que es una amenaza para las democracias, la pluralidad de pensamiento y la calidad de la información, considerándolo una fuente de desinformación por sesgo que debe ser detectada.

La crisis económica que comenzó en 2008 fue un duro golpe para los medios de comunicación, las agencias de noticias y la publicidad en general. En España entre 2008 y 2013 se cerraron un total de 284 medios debido a que la inversión publicitaria había decaído más del 50\% generando pérdidas económicas y eliminando 11.151 puestos de trabajo (Asociación de la Prensa de Madrid, 2013). Como consecuencia de esta decaída, los medios optaron por reducir los costos de producción, disminuyendo su tirada y producción propia, prescindiendo de sus trabajadores en el extranjero corresponsalías-, acudiendo a agencias internacionales de noticias y emigrando a las plataformas digitales, lo cual afectó fuertemente a la calidad informativa y resultado de las noticias finales de los medios (De-Pablos \& Mateos-Martín, 2004). 


\section{Civila, S., Castillo-Abdul, B., y Romero-Rodríguez, L. M. \\ El «efecto ventrilocuo» en las agencias internacionales de noticias. Revisión teórica e incidencia en las nuevas formas de desinformación}

Debido al apoyo de los medios de comunicación en las agencias internacionales de noticias durante todo este periodo, resulta fundamental destacar el rol actual que tienen las agencias de noticias y las cadenas transnacionales en el oligopolio informativo de asuntos que van más allá de las fronteras nacionales. Los cambios que están sufriendo los medios de comunicación convencionales, como son la prensa, la radio y la televisión, compiten en el ecosistema informativo con las redes sociales y los medios digitales. Esta situación ha forzado a los medios de comunicación nacionales a disminuir los costes de producción informativa (De-Pablos \& Mateos-Martín, 2004), lo que aumenta la dependencia a las cadenas internacionales de noticias $-v . g r$. CNN, $\mathrm{BBC}, \mathrm{Al}$ Jazeera, entre otras- y agencias internacionales -v.gr. Reuters, AP, AFP, EFE, etc.-. Aspinall (2005) señala que los medios nacionales tienden a tener un amplio número de periodistas desplazados a lugares clave dentro de un mismo territorio, aunque la situación cambia cuando se trata de informar sobre asuntos exteriores, ya que para tales labores los periodistas parecen incapaces de cubrir las noticias de forma personal, bien por la ausencia de apoyos para la cobertura fuera de sus fronteras, $\mathrm{o}$ bien por los propios recortes de personal.

Esto origina de alguna manera un «efecto ventrílocuo», en el que una sola o pocas perspectivas informativas se aceptan como ciertas por parte de todos los medios de comunicación, debido a la insuficiencia que tiene el sistema mediático de corroborar los hechos con periodistas en el lugar de los hechos (Arráez, 1998). Este fenómeno también podría producirse porque los diferentes medios, como afirma Rodgers (2003, p. 6), "se nutren de las mismas fuentes". Otros autores, como Arroyo y Yus (2007) tampoco dudan en afirmar que la mayoría de lo que leemos en un periódico proviene de la información que las agencias de noticias proporcionan.

Por su parte, Cooper (1957) se refería a este fenómeno en el meridiano del siglo XX, cuando explicaba cómo, en una boda, la novia le preguntó a qué se dedicaba: “ ¿Ha oído hablar de Associated Press?", a lo que la novia respondió “¡Por supuesto! -exclamó la novia- Mi marido siempre la lee. En todos los periódicos". Por tanto, se puede decir que son estas agencias y medios transnacionales los que determinan la agenda setting, el encuadre y sus diferentes versiones, generando las matrices de opinión de cualquier acontecimiento. A pesar de que aparentemente exista «pluralidad de sesgos», en realidad no es más que el resultado de la paráfrasis de estos -como caja de resonanciaen cada medio o en las propias informaciones que se comparten a través de las redes sociales. Esto es exactamente lo que provoca el "efecto ventrílocuo», que se puede resumir como "múltiples medios, una sola voz" (Arraez, 1998).

[...] esto podría dar lugar a la unificación de criterios informativos y de programación, aun cuando las líneas editoriales parecieren disímiles, por lo que la multiplicidad de canales $u$ opciones no debe determinar el pluralismo informativo, ideológico o de la libertad de expresión, sino que pudiera ser reflejo directo de una mayor concentración empresarial de la titularidad de esos medios. Esta es, a la vez, ilegítima por sus efectos, debido al ejercicio continuo de un poder lineal descendente que busca que los receptores actúen de una manera distinta de cómo lo harían con una información veraz (Romero, 2011, p.8).

Vivat Academia. Revista de Comunicación. 2021, nº 154, 25-46 


\section{Civila, S., Castillo-Abdul, B., y Romero-Rodríguez, L. M. \\ El «efecto ventrilocuo» en las agencias internacionales de noticias. Revisión teórica e incidencia en las nuevas formas de desinformación}

En la misma línea, Noëlle-Neumann departía en el comienzo de la década de los 70 de la «consonancia» como uno de los orígenes de la uniformidad de las informaciones periodísticas (Dader, 2009). La «consonancia» representa la similitud entre las ideas, creencias y valores de los medios de comunicación, lo que explicaría cómo bajo un aparente modelo de pluralismo con multitud de cabeceras, se puede esconder una gran homogeneidad de temas (Noëlle Neumann, 1973).

Esta arista social tiene importancia en el campo académico, no sólo en el que se basa en las teorías críticas o marxistas de la comunicación de Adorno, Horkeimer, Marcuse o Habermas, sino también en el que trata el control, que avanza a un ritmo vertiginoso y el decrecimiento de la competencia por el flujo globalizador que crea como un oligopolio trasnacional de medios de comunicación, cada vez más centralizados en grandes conglomerados en el que las realidades se expresan de una sola forma de pensamiento, atentando contra la pluralidad (Rubido et al., 2009).

Según Gurevitch, Levy y Roeh (1991) y Clausen (2004), la cobertura de las noticias internacionales está sujeta a ciertos encuadres (frames), en el sentido que las agencias y medios internacionales mantienen inclinaciones culturales determinadas intentando enmarcar (framing), construir (agenda setting) y estimar-priorizar (priming) los acontecimientos desde el discurso dominante de sus audiencias, generalmente desde el enfoque occidental, donde el respeto por los Derechos Humanos, la democracia, el Estado de Derecho y la institucionalidad, son elementos concluyentes en la creación del discurso. A través del encuadre los medios conectan con las masas, definiendo la situación de un tema y estableciendo una posición para generar un debate. El framing permite identificar como los medios de comunicación abordan un hecho noticiable y a su vez determina la efectividad de la cobertura (Nwakpoke, et al., 2020).

Autores como Seaton (2003) se han interesado en el problema de la repetición de noticias, un hecho que, como se ha mencionado en referencia, proviene de un exceso de confianza o incluso dependencia de los diferentes medios con las agencias internacionales de noticias. Esta situación, cuyas consecuencias en el ámbito del pluralismo son tan evidentes como perniciosas, se intensifica si tenemos en cuenta otros aspectos derivados. En particular se van a resaltar 3 aspectos que pueden debilitar las informaciones enviadas al lector.

- Sesgo etnocéntrico.

Con sesgo etnocéntrico se hace referencia a que tanto las principales agencias de noticias, como sus audiencias, son en su generalidad occidentales, por lo que el framing de la noticia está orientado a una sola sociedad (Sanz, 2007). Como asegura Muro Benayas (2006), aunque la objetividad y el rigor son cualidades que forman parte de los valores de todas las agencias, su práctica está afiliada a una determinada cosmovisión -anglosajona, árabe, latinoamericana o asiática- que está por encima de la parcialidad de sus redactores (Muro Benayas, 2006). Esto incita a que con asiduidad

Vivat Academia. Revista de Comunicación. 2021, nº 154, 25-46 
Civila, S., Castillo-Abdul, B., y Romero-Rodríguez, L. M.

$E l$ «efecto ventrilocuo» en las agencias internacionales de noticias. Revisión teórica e incidencia en las nuevas formas de desinformación

las agencias ofrezcan un tipo de cobertura diferente de sucesos similares, según en qué parte del globo sucedan (Branston \& Stafford, 2003).

- Otras fuentes

Las agencias de noticias internacionales no tienden a asegurar que sus periodistas tienen la capacidad de presenciar todos aquellos hechos sobre los que escriben (Fenby, 1978). Es más, cuando la realidad noticiable les sobrepasa, utilizan el recurso de personas que sí están en disposición de saber lo que ha ocurrido. Se puede mencionar como ejemplo el caso de la revista Der Spiegel, donde uno de sus periodistas ha escrito noticias manipuladas basadas en datos tomadas de otros medios y de imágenes de películas.

Al respecto, Tuchman (1978) manifiesta que los profesionales de la comunicación utilizan las citas como protección para alejarse de los acontecimientos. Este proceso de traspaso de responsabilidad y accountability no tiene por qué acabarse aquí (Bishop, 2001); por ello no resulta extraño que en un proceso casi de continua paráfrasis, el lector final de una noticia pueda convertirse, en realidad, en el último eslabón de una cadena de fuentes que remiten a fuentes que, a su vez, remiten a otras fuentes.

Las cadenas de televisión y radio, o incluso los portales de Internet (Laso \& Iglesias, 2002) parecen estar regidos aún más por las limitaciones derivadas de la intensa competitividad y del deseo exacerbado de inmediatez, así que la dependencia de las agencias puede darse, incluso, con mayor intensidad (Domingo Santamaría, 2006).

\section{- Efecto agenda setting}

Las agencias internacionales de noticias mantienen un sistema teledirigido, lo que tiene como consecuencia que la audiencia, cada vez más elevada, esté expuesta a una agenda de noticias cada vez más disminuida (Gelado, 2009). Por tanto, si hay menos «voces» diciéndole al resto de los medios qué es noticia y qué no, hay más posibilidades de que haya un mayor número de hechos que queden fuera de la agenda informativa (priming que genera el gatekeeping y en derivación, la omisión volitiva y la censura). Como consecuencia, parece que la realidad es que solo una pequeña cantidad de hechos pasan a ser noticias reseñadas (Hall, 1973).

Por su parte, la reiteración de los discursos en cada uno de los medios no hace más que reducir el pluralismo, en tanto que hace que el número de publicaciones pase a no ser importante, ya que lo que importa al final es el número de agencias que proveen de información a los medios (Gelado, 2009). De acuerdo con el mismo autor (op. cit., 2009), tal repetición contribuye al efecto agenda setting y produce, además, que no sólo se repitan temas, sino que se sostengan también estereotipos. 
Civila, S., Castillo-Abdul, B., y Romero-Rodríguez, L. M.

El «efecto ventrilocuo» en las agencias internacionales de noticias. Revisión teórica e incidencia en las nuevas formas de desinformación

\subsection{Desinformación, «efecto ventrílocuo» y medios digitales}

Internet ha significado con su auge y popularización no solo aspectos positivos, si no también problemáticas como la sobresaturación y la infoxicación, de las cuales se hablará más adelante. Esto ha provocado un especial cambio en la forma de consumo de información y la crisis de los medios convencionales (Shenk, 2003). La aparición de las redes sociales y las plataformas digitales traen consigo características como la inmediatez, la omnipresencia y ubicuidad, la interacción y en muchos casos, la gratuidad, presionando a todos los medios a compartir estas características, lo que hace complicado distinguir entre información, rumores y desinformación. De acuerdo con López-García (2004), esta situación conduce a abandonar los medios tradicionales como principal fuente de información.

Los editores de contenido en Internet producen sus propias informaciones periodísticas, pero la investigación en el contenido de noticias en el ámbito digital demuestra que generalmente lo mezclan con una gran cantidad de contenido de productores secundarios, como los servicios push, normalmente proporcionados por los principales portales de periodismo electrónico que brindan un flujo continuo de contenido y de informaciones.

Estos servicios en Internet originan sus propioss contenidos a raíz de las agencias internacionales de noticias sin cambios o apenas modificaciones. Por tanto, las agencias llegan a la audiencia bien a través de sus propios sitios web, como de todos los demás proveedores importantes de noticias en línea (como Google News). Aunque las agencias de noticias han tratado históricamente de minimizar su exposición pública, ahora dependen del atractivo popular y de sus nombres de marca para la audiencia del mundo digital, por lo que ahora comercializan sus nombres agresivamente en el sector del «periodismo electrónico». La información proporcionada, sin embargo, rara vez proviene de agencias de noticias diferentes a $A P$ o Reuters.

Los conglomerados globales de información multimedia, como Associated Press (AP) y Reuters, dominan las noticias de Internet en su mayoría de manera discreta, y su superioridad parece estar creciendo. Miles de medios de comunicación de todo el mundo realizan informes originales sobre historias masivas ocasionales, como la guerra, pero la cobertura internacional, en la mayoría de los demás casos, se deja casi exclusivamente a las agencias de noticias (Torres Toukoumidis et al., 2017).

Las manifestaciones de convergencia han dado lugar a un sector de periodismo electrónico en el que la producción de información sobre asuntos públicos está más concentrada de lo que ya estaba. Dichas tendencias indican que una infraestructura de información global que contradiga el pensamiento y dogma popular va a ser indiferente e incapaz de desafiar las desigualdades existentes. Lo que viene a ocurrir entonces con la concentración multimedia es una gran cantidad de efectos secundarios, propios del ecosistema comunicativo actual, y que provoca el ya mencionado anteriormente «efecto ventrílocuo».

Vivat Academia. Revista de Comunicación. 2021, nº 154, 25-46 
Civila, S., Castillo-Abdul, B., y Romero-Rodríguez, L. M.

El «efecto ventrilocuo» en las agencias internacionales de noticias. Revisión teórica e incidencia en las nuevas formas de desinformación

Tal y como se extrae de lo anterior, como todo cambio de sistema, la masificación de Internet ha aproximado a nuevos problemas, los cuales han producido diferentes consecuencias:

- Sobresaturación e infoxicación del espectro comunicativo (v. gr. Shenk, 2003, p. 396-397; Cornellà, 2010; Carr, 2011). Esto se debe al crecimiento y la importancia que han adquirido las redes sociales, ya que se han convertido en un repositorio de información y de medio de intercambio de esta.

- La distribución descentralizada de fake news (v. gr. Lotero-Echeverri, RomeroRodríguez \& Pérez-Rodríguez, 2018; Sartori, 1998; Ortega, 2006), que, aunque siempre ha existido en medios convencionales, a través de Internet alcanzan un gran impacto y viralidad (Pauner, 2018). Las noticias falsas, de acuerdo con Quandt et al., (2020), se construyen a través de la falsificación de los hechos, errores accidentales, negligencias y manipulación intencionada.

- Aumento de la crisis de los medios convencionales que ha fomentado la reducción de plantilla de periodistas (v. gr. Thom, 1992, p.14; López-García, 2004, p. 23).

- La multiplicación de informaciones sin verificación, lo que produce un efecto desinformativo (misinformation). El término desinformación se usa para hacer referencia a la intención de manipular a las personas a través de información deshonesta (Del Hoyo, et al., 2020)

- El impacto que los bots automáticos y los algoritmos de las redes sociales han tenido en la población, segregando la información a los gustos e intereses de cada usuario (Frost, 2019; Tejedor-Calvo et al., 2020).

Estos aspectos pueden constituir un escenario perfecto para la desinformación estructural en el ecosistema, teniendo un alcance insospechado y para el que ninguna institución social estaba preparada (v.gr. Pérez-Rodríguez, 2003; Pérez-Tornero, 2005; Romero-Rodríguez, Valle Razo \& Torres-Toukoumidis, 2018; Lotero-Echeverri, Romero-Rodríguez \& Pérez-Rodríguez, 2018).

\section{CONCLUSIONES Y DISCUSIÓN}

Tal como expone la revisión ejecutada, las principales agencias de noticias son las encargadas de divulgar la información por el resto de los medios, provocando el denominado «efecto ventrílocuo». Este resultado surge a partir del exceso de información reincidente en los canales de difusión. En consecuencia, existen noticias de interés global que quedan fuera del proceso de divulgación (omisión) y por lo tanto, fuera del espectro informativo.

A pesar de que algunos autores, como Cooper (1957), ya hablaban de este fenómeno y de cómo la falsa pluralidad afectaba a las sociedades, ofreciendo visiones estereotipadas e impidiendo la irrupción de nuevos o diferentes enfoques en la opinión pública, es un efecto que sigue generando impactos negativos en la sociedad. Además, con el desarrollo de las redes sociales, el «efecto ventrílocuo» se está magnificando por 
Civila, S., Castillo-Abdul, B., y Romero-Rodríguez, L. M.

El «efecto ventrilocuo» en las agencias internacionales de noticias. Revisión teórica e incidencia en las nuevas formas de desinformación

el alcance que en ellas se genera con respecto a la lectura de usuarios y la pluralidad de información en Internet.

Además, de acuerdo con Frost (2019) se observa cómo los bots automáticos y los algoritmos de Internet contribuyen aún más a la propagación de este efecto, provocando la segregación de noticias según los gustos del receptor. En cambio, otros autores como Zhang et al., (2020) defienden que con los múltiples medios independientes que surgen en Internet el efecto ventrílocuo disminuye, aunque el número de noticias falsas y desinformaciones que se viralizan están, lógicamente, en aumento debido a la descentralización de contenidos.

No obstante, la sobreutilización de las agencias de noticias y, por lo tanto, el continuo desarrollo del «efecto ventrílocuo» tiene consecuencias notorias en el mundo de la comunicación, así como en las sociedades. Tal como se ha puesto de manifiesto anteriormente, algunas de las consecuencias de este efecto es la desinformación, aquellas noticias no verificadas o con intención de engañar que están en Internet y divulgadas por las redes sociales, con el fin de movilizar la opinión pública hacia un punto en concreto o de lucrarse de ingresos publicitarios. En segundo lugar, está la sobresaturación informativa, que produce confusión debido a la dificultad de los seres humanos de procesar una gran cantidad de información.

La relación entre la desinformación y el «efecto ventrílocuo» es estrecha. La multiplicación de canales producido por el auge de las tecnologías ofrece una «falsa sensación de información». Si, se considera la desinformación como una falta de información y se selecciona la definición de Arraez (1998) sobre el «efecto ventrílocuo»: múltiples medios, una sola voz, se puede llegar a la conclusión de que una sola noticia divulgada por varios medios no conduce a la información plural y de calidad, sino a un estado de embriaguez donde todo es confuso, poco claro y limitado por las consecuencias de este efecto.

El «efecto ventrílocuo» nos dirige a la desinformación, ya que la realidad es que no se informa de todos los hechos que podrían ser noticiables, ni se ofrecen diferentes perspectivas de los que si llegan a estar en la agenda setting.

De acuerdo con Civila et.al., (2020) para evitar este tipo de daños sociales es importante la instauración de la educomunicación y alfabetización mediática en los planes educativos y sociales, con el fin de que la población interprete y comprenda el funcionamiento de los medios de comunicación.

La educomunicación supone un reto en la social actual, pues el desarrollo de habilidades críticas en el entorno digital permite interactuar con la información de forma responsable y ética (Area, 2012). En múltiples estudios, se evidencia que la ampliación de las habilidades digitales es fundamental para actuar críticamente en la sociedad. El tiempo que se pasa en Internet y el nivel de alfabetización mediática condiciona la actitud social hacia los hechos noticiables (Ayoub \& Garretson, 2017; Robinson \& Martin, 2009). 
Civila, S., Castillo-Abdul, B., y Romero-Rodríguez, L. M.

El «efecto ventrilocuo» en las agencias internacionales de noticias. Revisión teórica e incidencia en las nuevas formas de desinformación

La falta de pluralismo en los medios de comunicación, definitivamente, puede ser un objeto de estudio clave en el campo de la educomunicación, pues es un fenómeno que puede afectar a la percepción de los ciudadanos sobre ciertos temas.

En definitiva, el concepto del «efecto ventrílocuo» ha sufrido una transformación producto de los años, los cambios en el comportamiento del consumidor (ahora prosumidor) y del surgimiento de nuevos canales y espacios digitales de divulgación.

En principio, la literatura hace referencia a las agencias de noticias y a la compra de la información por parte de otros medios. Es decir, todos los medios disponibles se nutrían de las mismas fuentes. Por el contrario, en la actualidad se habla de este efecto como un arma de desinformación que provoca la falta de difusión de otras noticias relevantes, basadas en los algoritmos que se producen en los espacios digitales, producto de las lecturas e interacciones que reciben. Se prevé que, a mayor número de usuarios activos en redes sociales, la posibilidad de incrementar el efecto será superior.

\section{REFERENCIAS}

Agudelo Builes, I. (1996). La influencia de las rutinas productivas en el acontecer nacional. El caso de Siglo 21, periódico de Guadalajara. Comunicación y Sociedad, 28, 83-113.

Ayoub, P., \& Garretson, J. (2017). Getting the message out: Media context and global changes in attitudes toward homosexuality. Comparative political studies, 50(8), 1055 1085. https:// doi.org/10.1177/0010414016666836

Área, M. (2012). De lo sólido a lo líquido: las nuevas alfabetizaciones ante los cambios culturales de la Web 2.0. Comunicar, 19(38), 13-20. https://doi.org/10.3916/C38$\underline{\text { 2011-02-01 }}$

Arroyo, L. y Magali, Y. (2007). Los cien errores de la comunicación de las organizaciones. ESIC Editorial: Madrid.

Arráez, R. (1998). La comunicación para el desarrollo. Su aplicación para el medio radio. Revista Latina de Comunicación Social, 9(1), s/p. http://www.revistalatinacs.org/a/40rosa.htm

Aspinall, C. (2005). The News Monopoly. Free Press. Journal of the Campaign for Press and Broadcasting Freedom, 144, 1-2.

Bishop, R. (2001). News Media Heal Thyselves: Sourcing Patterns in News Stories about News Media Performance. Journal of Communication Inquiry, 25(1), 22-37. https://doi.org/10.1177/0196859901025001003 
Civila, S., Castillo-Abdul, B., y Romero-Rodríguez, L. M.

El «efecto ventrilocuo» en las agencias internacionales de noticias. Revisión teórica e incidencia en las nuevas formas de desinformación

Bruns, A., Highfield, T., Burgess, J. (2013). The Arab Spring and social media audiences: English and Arabic Twitter users and their networks. American Behavioral Scientist, 57(7), 871-898. https://doi.org/10.1177/0002764213479374

Branston, G., y Stafford, R. (2003). The media student's book. Routledge: London.

Camacho Domínguez, M. A., Romero-Rodríguez, L. M. \& Aguaded, I. (2018). Lucha de civilizaciones: la integración de los musulmanes en occidente. Espacios, 39(6), 917.

Civila, S., Romero-Rodriguez, L. M., \& Aguaded, I. (2020). Competencia mediática contra el odio, la violencia discursiva y la confrontación: Análisis documental y de teoría fundamentada. Temas de comunicación, 41, 93-109.

Chen, Y., Conroy, N., y Rubin, V. (2015). News in an online world: The need for an 'automatic crap detector. In: Procs of the Association for Information Science and Technology, 52(1), 1-4. https://doi.org/10.1002/pra2.2015.145052010081

Chomsky, N. y Herman, E. (1990). Los guardianes de la libertad. Barcelona: Grijalbo.

Cooper, K. (1957). Kent Cooper and the Associated Press: An Autobiography. Random Press: New York.

Clausen, L. (2004). Localizing the global: Domestication'processes in international news production. Media, Culture $\mathcal{E}$ Society, 26(1), 25-44. https://doi.org/10.1177/0163443704038203

Dader, J. L. (2009). Los cinco jinetes apocalípticos del periodismo español. Concentración, consonancia, constricción, clausura y comercialidad. Sala de Prensa, $6(3)$.

Day, A. (2018). Throwing our voices: ventriloquism as new media activism. Media, Culture E Society, 40, 617-628. htps://doi.org/10.1177/0163443718756064

De Bustos, M. (1993). Los grupos multimedia: Estructuras y estrategias en los medios europeos. Barcelona: Bosch.

De Bustos, M. y Pastor, F. (1995). El sector de las agencias de noticias en España. Análisis de su estructura y sus debilidades. Telos, 43, 25-32. http://www.quadernsdigitals.net/datos_web/hemeroteca/r_32/nr_467/a_6319/ $\underline{6319 . h t m l}$

Del Hoyo-Hurtado, M., García-Galera, M. C. \& Blanco-Alfonso, I. (2020). Desinformación y erosión de la credibilidad periodística en el contexto de las noticias falsas. Estudio de caso. Estudios sobre el Mensaje Periodístico, 26(4), 1399-1409. http://dx.doi.org/10.5209/esmp.70238

Vivat Academia. Revista de Comunicación. 2021, n 154, 25-46 
Civila, S., Castillo-Abdul, B., y Romero-Rodríguez, L. M.

El «efecto ventrilocuo» en las agencias internacionales de noticias. Revisión teórica e incidencia en las nuevas formas de desinformación

Doob, L. (1950). Goebbels' Principles of Propaganda. The Public Opinion Quarterly, $14(3), 419-442$.

Domingo Santamaria, D. (2006). Inventing online journalism. Development of the Internet as a news medium in four Catalan online newsrooms (Tesis doctoral). Universitat Rovira i Virgili, Tarragona.

Dretske, F. (1981). Sensation and Perception. Cambridge: MIT Press.

Fenby, J. (1986). The international news services: A twentieth century fund report. Schocken Books: New York.

Frické, M. (1997). What are the advantages of hyperproof-Like reasoning systems? British Journal for the Philosophy of Science, 48(3), 397-410. https://doi.org/10.1093/bjps/48.3.397

Frost, C. (2019). The power of voice: bots, democracy and the problem of political ventriloquism. Journal of Political Power, 13(1), 6-21. https://doi.org/10.1080/2158379X.2019.1701831

Gelado, R. M. (2009). El papel de las agencias de prensa españolas dentro del panorama mediático nacional. Comunicación y pluralismo, 8, 97-114.

https://doi.org/10.36576/summa.29191

Gonzalo Sanz, L. (2007). Entre Libertad y Determinismo. Genes, cerebro y ambiente en la conducta humana. Ediciones Cristiandad: Madrid.

Gurevitch, M., Levy, M. \& Roeh, I. (1991). The global newsroom: Convergences and diversities in the globalization of television news. En P. Dahlgren y C. Spark (Ed.), Communication and citizenship: Journalism and the public sphere (pp.195-216). New York, Estados Unidos: Routledge.

Grice, P. (1989). Studies in the way of words. Boston: Harvard University Press.

Hall, S. (1973). The determination of News Photographs. Sage: Beverly Hills.

Jiménez, G. (2011). Las agencias de noticias: Estructura de la información en Andalucía, 387 - 409.

Laso, I. e Iglesias, M. (2002). Internet, comercio colaborativo y mComercio: Nuevos modelos de negocio. Mundi-Prensa Libros: Madrid.

López García, G. (2006). Comunicación digital y líneas de fractura en el paradigma de la agenda setting. Doxa Comunicación, 4, 37-58. 
Civila, S., Castillo-Abdul, B., y Romero-Rodríguez, L. M.

El «efecto ventrilocuo» en las agencias internacionales de noticias. Revisión teórica e incidencia en las nuevas formas de desinformación

López, A. (2004). La desinformación en la aldea global como forma de conocimiento. Ágora Revista de Ciencias Sociales, 10, 19-30.

Lotero-Echeverri, G., Romero-Rodríguez, L. M. \& Pérez-Rodríguez, M. A. (2018). 'Factchecking' vs. 'Fakenews': Periodismo de confirmación como recurso de la competencia mediática contra la desinformación. Index. Comunicación, 8(2), 295-316.

Nwakpoke Ogbodo, J., Chike Onwe, E., Chukwu, J., Jude Nwasum, C., Sanita Nwakpu, E., Ugochukwu Nwankwo, S., Nwamini, S., Elem, S., \& Iroabuchi Ogbaeja, N. (2020). Communicating health crisis: a content analysis of global media framing of COVID-19. Health Promot Perspect, 10(3), 257-269.

Mas de Xáxas, X. (2005). Mentiras: Viaje de un periodista a la desinformación. Destino: Barcelona.

Muro Benayas, I. (2006). Globalización de la información y agencias de noticias: entre el negocio y el interés general. Barcelona: Paidós.

Noëlle-Neumann, E. (1973). Return to the Concept of Powerful Mass Media. Studies of Broadcasting, 9, 67-112.

Noëlle-Neumann, E. (1987). The 'event as event' and the 'event as news': The significance of consonance for media effects research. European Journal of Communication, 2, 391-414.

Noelle-Neumann, E. (1995). La espiral del silencio. Opinión Pública: Nuestra piel social. Barcelona: Paidós.

Ortega, F. (2006). Periodismo sin información. Madrid: Tecnos.

Pauner, C. (2018). Noticias falsas y libertad de expresión e información. el control de los contenidos informativos en la red. Teoría y Realidad Constitucional, 41,297-318.

Quandt, T., Frischlich, L., Boberg, S., \& Schatto-Eckrodt, T. (2020). Fake News. En T.P. Vos, F. Hanusch, D. Dimitrakopoulou, M. Geertsema-Sligh \& A. Sehl (Eds.), The International Encyclo-pedia of Journalism Studies. http://doi.org/10.1002/9781118841570.iejs0128

Reinares, F. (2012). Geografía mundial y del terrorismo. Madrid: Real Instituto El Cano.

Rivas Troitiño, J. (1995). Desinformación: Revisión de su significado. Del engaño a la falta de rigor. Estudios sobre el mensaje periodístico, 2, 75-83.

Rivas-Troitiño, J. M. (2001). Desinformación y Terrorismo: Análisis de las conversaciones entre el gobierno y ETA en Argel (enero-abril 1989) en tres diarios de Madrid [Tesis doctoral]. Madrid: Universidad Complutense de Madrid.

Vivat Academia. Revista de Comunicación. 2021, n 154, 25-46 
Civila, S., Castillo-Abdul, B., y Romero-Rodríguez, L. M.

El «efecto ventrilocuo» en las agencias internacionales de noticias. Revisión teórica e incidencia en las nuevas formas de desinformación

Rivera-Rogel, D., Zuluaga-Arias, L., Montoya Ramírez, N. M., Romero-Rodríguez, L. M., \& Aguaded, I. (2017). Media Competencies for the Citizenship Training of Teachers from Andean America: Colombia and Ecuador. Paidéia (Ribeirão Preto), 27(66), 80-89. https://doi.org/10.1590/1982-43272766201710

Rodríguez-Hidalgo, C., Rivera-Rogel, D., \& Romero-Rodríguez, L. M. (2020). Information Quality in Latin American Digital Native Media: Analysis Based on Structured Dimensions and Indicators. Media and Communication, 8(2), 135-145. http://dx.doi.org/10.17645/mac.v8i2.2657

Rodgers, J. (2003). Icons and invisibility: Gender myth. En Thussu, Daya Kishan y Friedman, Des (eds.), War and the media: reporting conflict 24/7. London: Sage.

Romero-Rodríguez, L. (2011). La manipulación informativa y la desinformación: La anomia de los receptores y el fomento de las víctimas propiciatorias. [Trabajo Fin de Máster]. Universidad de Almería.

Romero-Rodríguez, L. M., Valle Razo, A. L., \& Torres-Toukoumidis, A. (2018). Hacia una construcción conceptual de las Fake News: Epistemologías y Tipologías de las nuevas formas de desinformación. En: Pérez Serrano, M.J., Alcolea-Díaz, G. y Nogales-Bocio, A. I. (2018). Poder y medios en las sociedades del siglo XXI. Sevilla: Egregius Ediciones

Robinson, J. P., \& Martin, S. P. (2009). Social attitude differences between Internet users and non-uses: Evidence from the General Social Survey. Information, communication E society, 12(4), 508-524. https://doi.org/10.1080/13691180902857645

Rubido, S. (2009). Medios de Comunicación y Manipulación: Propuestas para una comunicación democrática. Madrid: Universidad Nacional de Educación a Distancia.

Sanz, L. (2007). Entre Libertad y Determinismo. Genes, cerebro y ambiente en la conducta humana. Ediciones Cristiandad: Madrid.

Seaton, J. (2003). Understanding not empathy. En Thussu, Daya Kishan y Friedman, Des (eds.), War and the media: reporting conflict 24/7. Sage: London.

Soengas, X. (2013). El papel de Internet y de las redes sociales en las revueltas árabes: Una alternativa a la censura de la prensa oficial. Comunicar, 4(21), 147-155. https:// doi.org/10.3916/c41-2013-14

Sönmez, S. (1998). Influence of terrorism risk on foreign tourism decisions. Tourism, Terrorism and political instability. Annals of Tourism Research, 25(2), 416-456. https://doi.org/10.1016/s0160-7383(97)00093-5 
Civila, S., Castillo-Abdul, B., y Romero-Rodríguez, L. M.

El «efecto ventrilocuo» en las agencias internacionales de noticias. Revisión teórica e incidencia en las nuevas formas de desinformación

Shenk, D. (2003). Information Overload, Concept of. En: E- Science, Encyclopedia of International Media and Communications, 2, 396. Boston: Elservier.

Tejedor-Calvo, S., Romero-Rodríguez, L. M., Moncada-Moncada, A. J., AlencarDornelles, M. (2020). Journalism that tells the future: possibilities and journalistic scenarios for augmented reality. El Profesional de la Información, 29(6). https://doi.org/10.3145/epi.2020.nov.02

Torres - Toukoumidis A., Romero-Rodriguez, LM., Casas-Moreno, P., y Aguaded, I. (2017). Construcción del discurso bélico desde las agencias internacionales de noticias: Estudio de caso atentados del 13 de noviembre de 2015. Revista mediterránea de comunicación, 8(1), 121-135. https:/ / doi.org/10.14198/MEDCOM2017.8.1.9.

Zhang, X., Ghorbani, A. A. (2020). An overview of online fake news: Characterization, detection, and discussion. Information Processing and Management, 57(2), 102025. https://doi.org/10.1016/j.ipm.2019.03.004

\section{AUTOR/ES:}

\section{Sabina Civila}

Investigadora Predoctoral FPI en el Departamento de Pedagogía de la Universidad de Huelva. Doctoranda en el programa Interuniversitario de Comunicación (UHU) en la línea de Educomunicación. Máster en comunicación estratégica e innovación de la comunicación (Málaga, Huelva, Sevilla y Cádiz), y Graduada en Publicidad y Relaciones Públicas por la Universidad de Cádiz. Investigadora del Grupo Investigación 'Ágora' (HUM-648). Actualmente enfoca su investigación a redes sociales, educomunicación, alfabetización mediática e islamofobia.

sabicivila@gmail.com

Orcid ID: $\underline{\text { https:/ / orcid.org/0000-0001-6059-9893 }}$

Google Scholar: $\underline{\text { https: / / scholar.google.es/citations?user=fZC19zMAAAAJ\&hl=es }}$

ResearchGate: https://www.researchgate.net/profile/Sabina_Civila 
Civila, S., Castillo-Abdul, B., y Romero-Rodríguez, L. M.

$E l$ «efecto ventrilocuo» en las agencias internacionales de noticias. Revisión teórica e incidencia en las nuevas formas de desinformación

\section{Bárbara Castillo-Abdul}

Doctoranda en el Programa Interuniversitario en Comunicación de las Universidades de Sevilla, Málaga, Huelva y Cádiz y Doctoranda del Programa en Ciencias Sociales y Jurídicas de la Rey Juan Carlos. Máster en Comunicación y Educación Audiovisual, itinerario de Investigación en Comunicación, por la Universidad Internacional de Andalucía y la Universidad de Huelva (España) y Premio Extraordinario por el trabajo de Investigación Fin de Máster otorgado por la Universidad de Huelva. Postgrado en Gerencia Social Media por la Universidad Católica Andrés Bello (Venezuela). Especialista universitario en Comunicación Organizacional por la Universidad Monteávila (Venezuela). Licenciada en Comunicación Social, mención Comunicación Corporativa por la Universidad Santa María (Venezuela). Profesora de ESERP Business School en Madrid y profesora visitante del ESAI Business School, Universidad Espíritu Santo en Ecuador. En el ámbito de investigación, posee un año de Estancia presencial Pre Doctoral en el TEC de Monterrey y tres meses de forma online. Miembro del equipo de trabajo del proyecto competitivo nacional I+D "Youtubers e Instagrammers: la competencia mediática en los prosumidores emergentes" (RTI2018-093303-B-I00), financiado por la Agencia Estatal de Investigación del Ministerio de Ciencia, Innovación y Universidades de España, así como del proyecto en Responsabilidad Social, Autorregulación Publicitaria y Salud en la Radio (RESAPS) (CSO2017-82267-R) de la Universidad Rey Juan Carlos.

barbaracastilloabdul@gmail.com

Orcid ID: https:// orcid.org/0000-0002-3711-1519

Google Scholar: https://scholar.google.es/citations?user=E74B2F0AAAAJ\&hl=es

ResearchGate: https://www.researchgate.net/profile/Barbara_Castillo-Abdul

\section{Luis Miguel Romero-Rodríguez}

Profesor del Departamento de Ciencias de la Comunicación y Sociología de la Universidad Rey Juan Carlos (Madrid, España). Doctor en Comunicación por las universidades de Huelva, Sevilla, Málaga y Cádiz. Máster en Comunicación Social por la Universidad de Almería y Licenciado en Periodismo por la Universidad Santa María (Venezuela). Editor asociado de la Revista Comunicar y de la Revista Anàlisi. Investigador Asociado del Grupo Ágora (PAI-HUM-648) de la Universidad de Huelva y miembro de la Red Interuniversitaria Euroamericana de Investigación en Competencias Mediáticas para la Ciudadanía (Alfamed). Su línea de investigación se enfoca en el estudio de la desinformación, el populismo y el control social, así como también en la Alfabetización Mediática y la competencia mediática de la ciudadanía.

Lromero2021@gmail.com

Orcid ID: https:/ / orcid.org/0000-0003-3924-1517

Google Scholar: https://scholar.google.es/citations?user=El_8FwoAAAAJ\&hl=es

ResearchGate: $\underline{\text { https:// www.researchgate.net/profile/Luis_Romero-Rodriguez2 }}$ 